\section{Analysis of High-Efficiency Solar Cells in Mobile Robot Applications}

\section{Francisco Calderón}

\section{Allan Lüders}

David Wettergreen

e-mail: dsw@ri.cmu.edu

\section{James Teza}

The Robotics Institute, Carnegie Mellon University, 5000 Forbes Avenue, Pittsburgh, PA 15213

\author{
Andrés Guesalaga ${ }^{1}$ \\ Department of Electrical Engineering, \\ Pontificia Universidad Católica de Chile, \\ 4860 Vicuna Mackenna, \\ Santiago RM 306-22, Chile \\ e-mail: aguesala@ing.puc.cl
}

This technical brief analyzes the performance of triple-junction solar cells on a mobile robot. Although originally designed for satellite use, it is demonstrated that triple-junction cells are effective in terrestrial applications. This makes them particularly suitable for systems with limited size and mass but high-power requirements such as a mobile robot. A testing station was specially constructed to characterize triple-junction and conventional silicon cell performance in different environments and to compare their effectiveness. Additional field tests were carried out with an autonomous robot in order to check the ability to deliver sufficient power to varying loads. Results show that they surpass conventional technologies with efficiencies higher than $22 \%$, so they can be considered as an alternative technology for power sources onboard of terrestrial mobile robots. [DOI: 10.1115/1.2735361]

Keywords: solar cells, advanced triple-junction, mobile robots

\section{Introduction}

It is well known that more efficient photovoltaic devices are needed for the development of market-competitive terrestrial solar powered mobile devices such as automobiles or robots, since silicon solar cells have efficiencies that are not high enough to achieve the power density required by these applications [1]. Decreasing costs of solar cells open a new range of possible applications for more efficient technologies such as those used in space where mass limitations dominate cost.

This technical brief presents an analysis of the electrical performance of commercial advanced triple-junction (ATJ) solar cells, built originally for space applications, and compares them to traditional technologies. We analyze the characteristics of ATJ solar cells under changing ambient conditions during a day, and also illustrate the importance of the solar incident angle on its perfor-

\footnotetext{
Corresponding author.

Contributed by the Solar Energy Division of ASME for publication in the JouRNAL of Solar Energy EngineEring. Manuscript received July 13, 2006; final manuscript received December 14, 2006. Review conducted by Antonio Marti Vega.
}

mance. In addition, we validate our experimental results by incorporating ATJ solar cells on a solar-powered rover to analyze performance improvements.

This work is part of the Life in the Atacama project, which seeks to develop technology in support of robotic astrobiology while conducting Earth science in the Atacama desert (Chile) [2]. Its purpose is to develop technology relevant to Martian expeditions in the form of an autonomous solar-powered rover, "Zoë" (Fig. 1). The robot is capable of traversing long distances to find basic forms of life without direct human intervention. The solar cell characterization experiment was carried out using a custombuilt testing station. The test station automatically adjusts both the solar panel orientation and its electrical load. It controls and tests two sets of solar cells simultaneously: ATJ and silicon, and also measures their temperature. The cells are mounted on a pointing platform controlled by a pan and tilt unit, that incorporates a 180 deg field of view (FOV) pyranometer and spectrophotometer that can measure solar flux in wavelength bands that cover the regions of sensitivity of the solar cells. The results obtained for the silicon solar cells are used as reference to assess the performance of the ATJ technology. The silicon reference units are the Siemens Standard Type 2 monocrystalline solar cells [3]. The ATJ structure is an $n / p \mathrm{InGa} / \mathrm{InGaAs} / \mathrm{Ge}$ solar cell manufactured by Emcore [4].

The experiments were carried out in the O'Higgins region (central Chile). All the data were acquired during a summer day, under clear skies. The experiments were repeated every $45 \mathrm{~min}$ from 11:00 am until 6:30 pm. The solar irradiance at the start and end of the experiments was of $856 \mathrm{~W} / \mathrm{m}^{2}$ and $446 \mathrm{~W} / \mathrm{m}^{2}$, respectively, with a peak of $1052 \mathrm{~W} / \mathrm{m}^{2}$ at $1: 51 \mathrm{p} . \mathrm{m}$.

\section{Results}

2.1 Performance Overview. Substantial differences exist in the performance of the two types of solar cells. ATJ technology operates at higher voltages and lower currents when compared to silicon cells (see Fig. 2). The efficiency of ATJ solar cells proved to be significantly higher, more than doubling silicon solar cells. This is reflected in the higher power output per area: $249 \mathrm{~W} / \mathrm{m}^{2}$ versus $111 \mathrm{~W} / \mathrm{m}^{2}$ for silicon devices, when the panels are positioned horizontally for the maximum solar irradiance episode of the day.

2.2 Performance Under Varying Conditions. Some factors affecting the performance of the solar cells fluctuate during the day. These include the spectral condition [5], solar incident angle (in cases where the panels remain in a fixed position), the ratio of diffuse/direct radiation, and the temperature of the solar cells, among others. To understand the effect of the varying external conditions on the solar cells, $I-V$ curves for different environmental conditions were captured.

The analysis of these technologies is based on direct sun radiation, i.e., diffuse radiation has not been considered during comparison. Previous reports [6] show that diffuse radiation can reach a fifth of the total radiation during clear skies in Mediterranean climates. However, this figure drops substantially when flat panel radiometry is considered. Furthermore, the diffusion radiation affects both technologies similarly, so efficiency results are still valid.

The solar radiation on a fixed surface will vary along the day (radiometric cosine projection), and also because the path length of the sun rays in the atmosphere changes according to the incident solar angle. These variations in path length cause changes in the solar spectra since the absorption in the atmosphere is wavelength dependent [7].

Figure 2(a) plots $I-V$ curves at different times when the panels are deployed horizontally. Large variations are observed in both the short-circuit current $\left(I_{\mathrm{sc}}\right)$ and open-circuit voltages $\left(V_{\mathrm{oc}}\right)$. Significant differences in current at low voltages are expected, since current values are proportional to the incident radiation, which is 


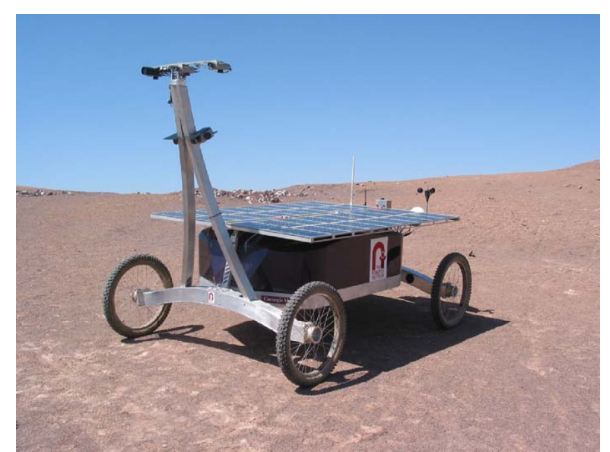

Fig. 1 Zoë robot during field operations in the Atacama desert, Chile

lowest during the early and late times of the day.

Current values are proportional to temperature and the opposite occurs with voltage. Table 1 shows significant variations in the measured operation temperature during the day, which unevenly affect the different experiments. In order to eliminate this effect, Fig. 2(b) presents temperature-corrected measurements $\left(28^{\circ} \mathrm{C}\right)$ using the correction factors provided by the manufacturer. Temperature-corrected measurements do not intersect, revealing that experiments carried out during high irradiance episodes are comparatively more degraded at higher temperatures.

A sun-tracking system has been implemented in order to maintain a normal orientation of the panels to the sun, aimed to increase radiometric area. Figure 3 shows temperature-corrected results for this system. Global performances of both solar cell technologies increase significantly, especially for small zenith
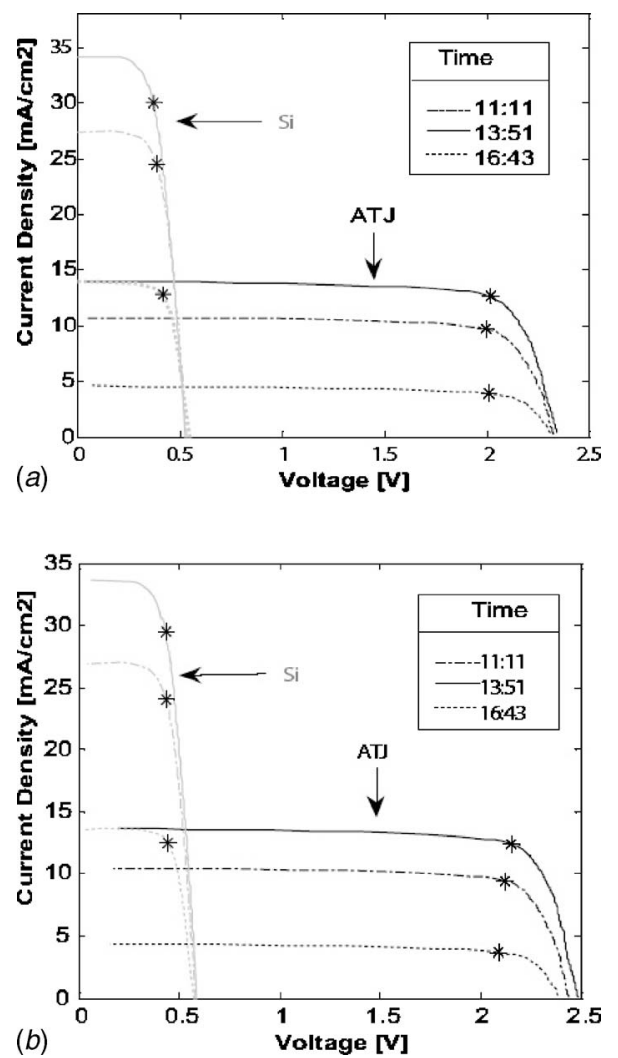

Fig. $2 I-V$ curves along the day for ATJ and silicon panels in horizontal positions: $\left(^{*}\right)$ maximum power conditions; (a) measured values; (b) temperature-compensated values to $28^{\circ} \mathrm{C}$
Table 1 Average temperatures in cells, horizontal position

\begin{tabular}{lcc}
\hline \hline Time & $\begin{array}{c}\text { ATJ } \\
\left({ }^{\circ} \mathrm{C}\right)\end{array}$ & $\begin{array}{c}\text { Silicon } \\
\left({ }^{\circ} \mathrm{C}\right)\end{array}$ \\
\hline $11: 11$ & 49.1 & 49.9 \\
$13: 51^{\mathrm{a}}$ & 53.7 & 56.7 \\
$16: 43$ & 52.8 & 53.7 \\
Average & 51.9 & 53.4 \\
\hline \hline
\end{tabular}

${ }^{\mathrm{a}}$ Maximum radiation time.

angles. As expected, the performance of the solar cells does not improve during the higher-irradiance episodes, since the sun is positioned almost normal to the solar cells in both cases.

2.2.1 Changes in Conversion Efficiency. Several additional experiments were carried out to determine the relationship among conversion efficiency, sun radiation conditions, and solar cell temperature. Figure 4 and Table 2 present variations in efficiency and other relevant parameters when cells are positioned horizontally. A significant variation in ATJ solar cells performance is observed; however, fill factors remain constant.

Solar cells were continuously served to four arbitrary orientations to determine the influence of the incident angle: horizontal (0 deg tilt); 34 deg and 44 deg north tilt; and directly toward the sun. Data obtained for a short period of time is used in order to isolate the experiment from changing environmental conditions (see Fig. 5). A significant performance falloff is noticed at increasing incident angles due to the higher sensitivity of multijunction devices to deviations from its nominal operating point. The reduction in performance of an individual layer propagates limiting the overall cell efficiency due to its serial configuration.

The decrease in performance for individual layers is possibly caused by variations in the reflection pattern and also by recombination in the layers. On the one hand, ATJ solar cells are built with broadband dual-layer coatings optimized to cancel reflections for the wavelengths of interest. For incidence angles different from nominal, the incoming beam finds an apparent thicker layer, and the effectiveness of the coating departs from its optimum. Considering that the top and middle layers in ATJ solar cells are direct band gap semiconductors, and that silicon is an indirect band gap material, an increased performance falloff is expected for the ATJ technology. Furthermore, the difference in performance is accentuated by the fact that the surface of typical commercial silicon cells has an inverted micropyramidal texture. This feature improves the absorption of light since it allows some of the reflected rays to impact the surface several times [8].

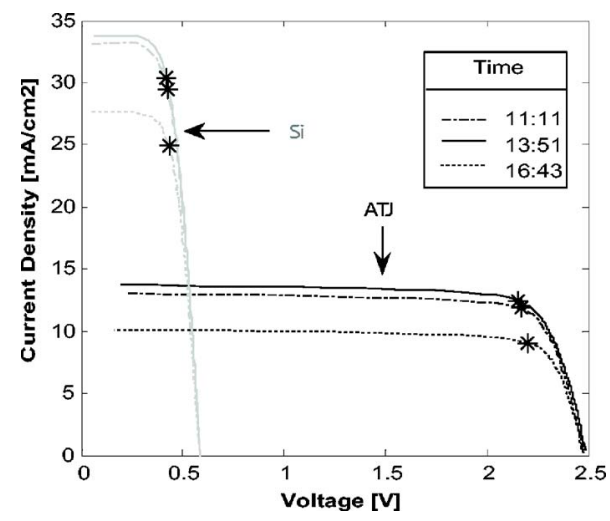

Fig. 3 Sun tracking $I-V$ curves for ATJ (left) and silicon (right); temperature compensation to $28^{\circ} \mathrm{C}$ 


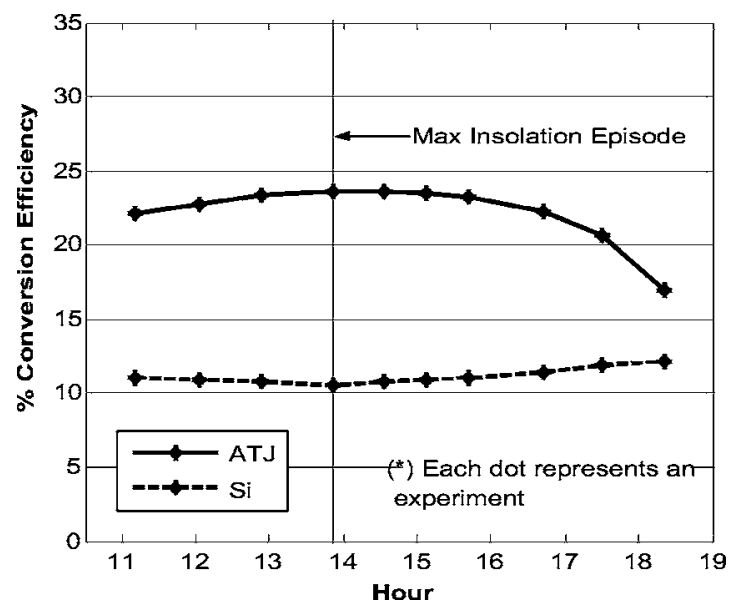

Fig. 4 Variations in conversion efficiency for temperaturecorrected data to $28^{\circ} \mathrm{C}$

\section{Applications in Robotics}

The results described in previous sections were verified in the field by incorporating state-of-the-art ATJ solar cells onboard the Zoë robot. Zoë is an autonomous Mars rover developed as part of the NASA astrobiology research program. The robot incorporates ATJ solar cells (deployed horizontally) to satisfy the power needs of the onboard equipment and instruments (four computers, pan and tilt unit, cameras, a spectrometer, and a fluorescence imager, among others). Zoë was tested during field operations in the Atacama desert (northern Chile) [2].

The rover relies on high-efficiency power-tracker circuits that can operate the array of solar cells at their maximum-efficiency power point when necessary. The operation point is set according to the instantaneous load and battery charging state.

In order to assess the efficiency of the system, solar irradiance is measured with a specially designed station deployed near the robot operating area.

It was found that Zoë's power tracker is able to achieve $22 \%$ efficiency with the ATJ solar cells (daily cycle base, average value) on a regular basis. This satisfies the current level required by Zoë that could not be achieved using silicon technologies.

Another variable of interest is the operational efficiency of the solar arrays, i.e., the instantaneous incident solar energy that is converted into useful electrical energy. As the solar cell might be operating in a suboptimal point depending of the amount of energy required by the rover, the operational efficiency will always be less than the actual conversion efficiency of the cells. Figure 6 shows the instantaneous operational efficiency of the solar cells and solar irradiance variation during a regular day of operation. Variations in efficiency along the day reflect changes in the load and the total power required by the rover. Steep drops correspond to extremely low-power requirements, e.g., when the rover is not moving and the batteries are already charged. The figure shows that the solar cells regularly work at $23 \%$ efficiency, slightly higher than the averaged results obtained by the ATJ cell analysis

Table 2 Summary of measured conversion efficiencies and fill factors

\begin{tabular}{lccc}
\hline \hline & & ATJ & Si \\
\hline Conversion efficiency & Average & $\mathbf{2 2 . 2}$ & $\mathbf{1 1 . 1}$ \\
$\boldsymbol{\eta} \%$ & Max & 23.6 & 12.1 \\
& Min & 17.0 & 10.6 \\
Fill Factor & Average & $\mathbf{7 7 . 2}$ & $\mathbf{6 3 . 4}$ \\
$\%$ & Std. dev. & 0.01 & 0.03 \\
\hline \hline
\end{tabular}

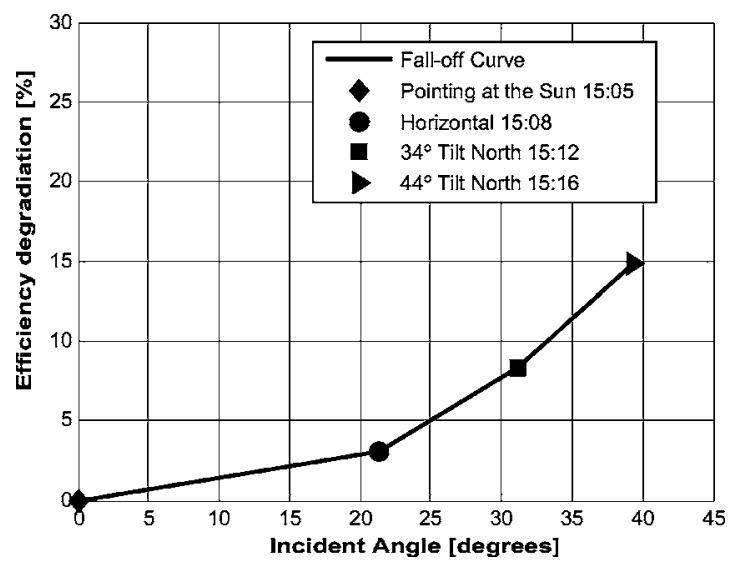

Fig. 5 Performance falloff due to increasing incidence angles for the ATJ solar cells (temperature-correction to $28^{\circ} \mathrm{C}$ )

carried out on the pointing platform. This can be explained by the difference in absorbance between the locations where the individual panels testing and rover trials took place.

\section{Conclusions}

ATJ InGaP/InGaAs/Ge solar cell technology, originally designed to operate in space, was tested for terrestrial operation on a mobile robot. An average efficiency of $22 \%$ was obtained in experiments carried out for individual cells, reaching a maximum of $23.6 \%$ for the most favorable conditions of the day (equivalent to $25 \%$ when corrected to $28{ }^{\circ} \mathrm{C}$ ). The results are significantly better than those obtained for conventional technologies, doubling the efficiency of silicon solar cells under equivalent conditions.

It was found that commercial ATJ cells present a variableefficiency characteristic with strong dependence on rays' incident angle. It was demonstrated that higher efficiencies can be obtained when the cell surface is positioned normal to the sun direction. This characteristic differs from commercial silicon technology (built for terrestrial use), presenting virtually no relevant efficiency deterioration with respect to incidence angle. A possible cause for the ATJ performance falloff might be the longer optical path in the antireflection coatings and thickness of semiconductor layers. The increase in the optical path length in the antireflection coating reduces the antireflection effectiveness, changing the reflectance distribution for which the semiconductor layer widths were designed. These factors do not affect silicon cells consider-

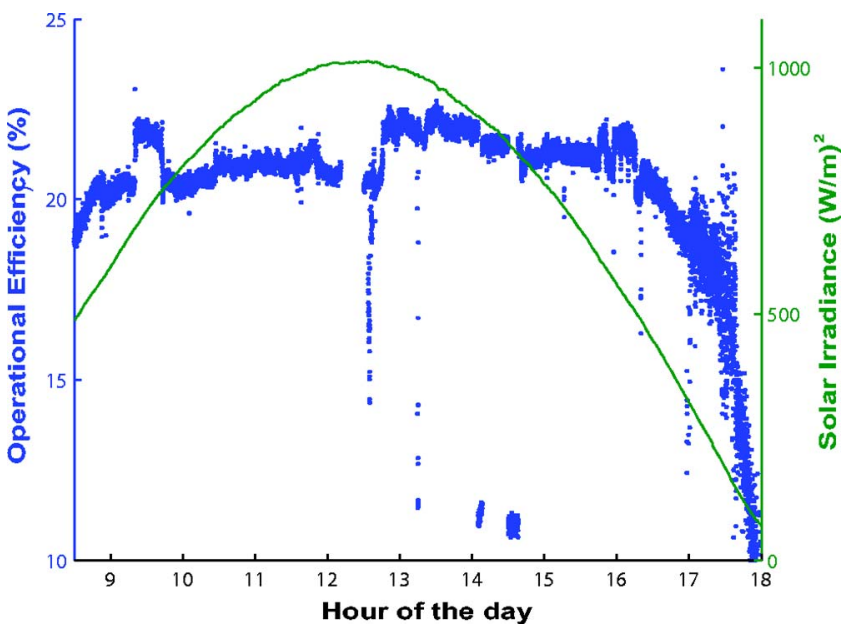

Fig. 6 Instantaneous solar array operation efficiency on Zoë 
ably, possibly due to their single-junction construction. Also, the presence of micropyramidal top surfaces allows them to capture light from wider angles of incidence. Additionally, silicon is an indirect-band gap material which is less susceptible to certain recombination types occurring more often in the direct-band gap materials of the ATJ.

Although the ATJ cells clearly outperformed silicon cells, further improvements can be achieved in this multijunction technology if terrestrial characteristics are considered during design, i.e., a better current matching strategy for the received spectrum, and also characteristics such as micropyramidal surfaces.

The technology was successfully tested onboard Zoë, showing that substantial increases in electrical load of autonomous vehicles can be handled by incorporating ATJ solar cells. Trials proved that it is possible to obtain efficiencies as high as $23 \%$ in a consistent and reliable manner, offering an alternative to traditional technologies.

Although ATJ cell efficiency is considerably better than in conventional technologies, economic issues may limit the introduction of ATJ solar technologies in the field. Nevertheless, they could become attractive when the vehicle's mass and volume design specs are restrictive.

\section{Acknowledgment}

This project was developed by Carnegie Mellon University with the collaboration of Pontificia Universidad Católica de Chile among other institutions, and it has been supported by NASA
Grant No. NAG5-12890. We thank Michael Wagner, Stuart Heys, and Christopher Williams for their help in the software design and during field experiments.

\section{References}

[1] Abusleme, A., Dixon, J., and Soto, A., 2003, "Improved Performance of a Battery Powered Electric Car, Using Photovoltaic Cells," Proceedings of the 2003 IEEE PowerTech Conference, Bologna, Italy, 23-26 June 2003.

[2] Wettergreen, D., Cabrol, N., Baskaran, V., Calderón, F., Heys, S., Jonak, D. Luders, R. A., Pane, D., Smith, T., Teza, J., Tompkins, P., Villa, D., Williams, C., and Wagner, M. D., 2005, "Second Experiments in the Robotic Investigation of Life in the Atacama Desert of Chile," Proceedings of the 8th International Symposium on Artificial Intelligence, Robotics and Automation in Space, Munich, Germany, 5-8 September 2005.

[3] Siemens Solar Industries, 1994, "High Efficiency Solar Electric Cells," Silicon Solar Cells Product Brief.

[4] ATJ Solar Cells, 2004, "Advanced Triple-Junction (ATJ) High Efficiency Solar Cells for Space Applications," ATJ Solar Cells Product Brief.

[5] Letay, G., Baur, C., and Bett, A. W., 2004, "Theoretical Investigations of III-V Multi-junction Concentrator Cells under Realistic Spectral Conditions," Proceedings of the 19th European Photovoltaic Solar Energy Conference, Paris, France, 7-11 June 2004.

[6] Psiloglou, B. E., Santamouris, M., and Asimakopoulos, D. N., 2000, "Atmosphere Broadband Model for Computation of Solar Radiation at the Earth's Surface. Application to Mediterranean Climate," Pure Appl. Geophys., 157, pp. 829-860.

[7] Wolfe, W. L., 1998, "Introduction to Radiometry," SPIE Tutorial Texts in Optical Engineering, Vol. TT29, SPIE Optical Engineering Press, Bellingham, WA.

[8] Al-Bustani, A., and Fetecha, M. Y., 1995, "Design of Antireflection Coatings for Triple Heterojunction AlGaAs-GaAs Space Solar Cells," Proceedings of the IEEE Workshop on High Performance Electron Devices for Microwave and Optoelectronic Applications, London, UK, 27-28 November 1995. 